\title{
MI TKA: A Risk Factor for Early Revision Surgery
}

\author{
Robert E. Mayle Jr., M.D. ${ }^{1}$ Bradley P. Graw, M.D. ${ }^{1}$ Heather G. Huddleston, M.D. ${ }^{2}$ \\ Steven T. Woolson, M.D. ${ }^{1}$ Stuart B. Goodman, M.D., Ph.D., F.R.C.S.C., F.A.C.S., F.B.S.E. ${ }^{1}$ \\ James I. Huddleston, M.D. ${ }^{1}$
}

${ }^{1}$ Department of Orthopaedic Surgery, Stanford University, Redwood City, California

${ }^{2}$ Department of Obstetrics, Gynecology, and Reproductive Science, University of California San Francisco, San Francisco, California

\begin{abstract}
Address for correspondence and reprint requests Robert Edward Mayle, Jr., M.D., Department of Orthopaedic Surgery, Stanford University, 450 Broadway Street, MC 6342, Redwood City, CA 94063
\end{abstract} (e-mail: robmayle@gmail.com).

J Knee Surg 2012;25:423-428.

\begin{abstract}
Keywords

- minimally invasive total knee arthroplasty

- failed total knee arthroplasty

- revision total knee arthroplasty

Minimal incision total knee arthroplasty (MI TKA) was developed with the potential to decrease surgical trauma, pain, and recovery time. While this procedure has increased in popularity, some surgeons have questioned its safety and long-term efficacy. In this study 58 consecutive revision total knee arthroplasties (TKAs) (57 patients) performed at one academic medical center from 2006 to 2008 are reviewed. Prospectively collected clinical and radiographic data included: incision length, gender, age, time to revision surgery, and primary diagnosis at time of revision. Of these, 34 knees involving infection and rerevision were excluded. Of the remaining 24 knees, 11 knees that met inclusion criteria had undergone MI TKA. There were no differences between the groups with regard to age, diagnosis, body mass index, and gender. Average time to revision was shorter for the MI TKA patients ( 29 vs. 65 months, $p<0.032$, odds ratio 14.7). Reasons for revision were aseptic loosening (55\%), pain/stiffness (27\%), malrotation (9\%), and instability (9\%) in the MI TKA group and aseptic loosening (53\%), instability (15\%), pain/stiffness (8\%), malrotation (8\%), combined malrotation and instability (8\%), and polyethylene wear/osteolysis (8\%) in the traditional TKA group. These data suggest that MI TKA may be a risk factor for early revision.
\end{abstract}

Total knee arthroplasty (TKA) is one of the most successful surgical procedures performed with a $90 \%$ survival rate at 10 to 15 years. ${ }^{1}$ Effectiveness of relieving pain and restoring function after TKA is undisputed. ${ }^{2}$ Long-term implant performance and low revision rates have historically defined success in TKA, with less emphasis on the length of the incision, length of hospital stay, duration of dependence on an assistive devices, and length of time to complete recovery. ${ }^{3}$

Despite the evolution of patient selection, implants, and instrumentation to improve the effectiveness of TKA, numerous studies show that patient satisfaction following TKA is between 75 and $89 \% .{ }^{4-8}$ These studies signify a discrepancy between the objective-physician and subjective-patient as- sessment following TKA. Bullens et $\mathrm{al}^{2}$ note that while physicians tend to focus on range of motion (ROM), alignment, and stability, patients focus on the overall functionality of the knee. These studies indicate that there is a need to align physician and patient outcome measures and that there is room for improvement in functional outcomes following TKA.

Repicci and Eberle ${ }^{9}$ introduced the concept of minimally invasive (MI) knee surgery with regards to unicondylar knee arthroplasty (UKA) in the 1990s. Their approach used special instruments, a smaller surgical incision, and avoidance of normal soft tissue sacrifice (opposite compartment, cruciates, and patellar bone) and disruption of the patellofemoral articulation. Successful application of this technique led to received

December 11, 2011

accepted

December 28, 2011

published online

May 15, 2012
Copyright $\odot 2012$ by Thieme Medical Publishers, Inc., 333 Seventh Avenue, New York, NY 10001, USA. Tel: +1(212) 584-4662.
DOI http://dx.doi.org/ 10.1055/s-0032-1313757. ISSN $1538-8506$. 
a resurgence in the popularity of UKA. ${ }^{10}$ With the improvement of surgical techniques and instrumentation, surgeons naturally questioned if similar principals could be applied to TKA. ${ }^{11}$ While there is no universally accepted definition for MI TKA, the concept of MI TKA encompasses a surgical approach to TKA through an incision length $<14 \mathrm{~cm}$, with limited surgical trauma by reduced soft tissue dissection, sparing of the quadriceps muscle, and avoidance of patellar eversion and tibiofemoral dislocation. ${ }^{12,13}$

Proponents for MI TKA advocate higher level of patient satisfaction, earlier return of function, earlier independent straight leg raise, less need for assistive devices, an increase in short-term ROM, less need for inpatient rehabilitation postdischarge, less postoperative pain and narcotic use, shorter length of hospital stay, and increased cost-effectiveness. $^{10,14-17}$ Despite this, there is a concern over the widespread promotion and application of MI principles to TKA, specifically regarding complications and long-term outcomes. $^{18}$ Barrack et al $^{10}$ recently published a study based on the experience of five surgeons at three referral centers. They noted a statistically significant shorter time to revision for patients who had undergone MI TKA compared with traditional TKA (14.8 vs. 80 months, $p<0.001$ ).

While the intention of MI TKA to decrease postoperative pain and improve the return of postoperative function is valid, the evidence surrounding the short-term benefit of MI TKA is limited, and there is sparse evidence concerning the long-term safety and efficacy of these operations. At our referral center, we noted an increasing percentage of revision TKA cases associated with small incisions. In this study, we compared our patients requiring revision TKA who had undergone MI TKA as their index procedure with those patients who had undergone their primary index TKA through a traditional approach. We hypothesized that there would be no difference between the two groups regarding the time from the index procedure to the revision procedure.

\section{Materials and Methods}

A consecutive series of revision TKAs performed at one academic medical center from 2006 to 2008 was retrospectively reviewed with institutional review board approval. During this period, 58 revision TKAs were performed in 57 patients by a single surgeon. Patients with surgical incisions $<11 \mathrm{~cm}$ in length were included in the MI group. All of the index MI procedures were performed at several outside institutions by multiple different surgeons. Of the 58 revision TKAs performed, 15 met the criteria for MI TKA, leaving 43 as non-MI TKA. Infections and rerevisions were excluded, leaving 24 knees that met our inclusion criteria (11 MI TKA vs. 13 traditional TKA). The number of females was $7 / 11$ patients in the MI TKA group and 5/13 patients in the traditional TKA group. A detailed clinical analysis included review of the initial patient consultation notes, earlier medical records, clinical progress notes, radiographs, and the operative report of the revision procedure. Data obtained on every patient included age at the index procedure, primary diagnosis before index procedure, time to revision, gender, body mass index (BMI), reason for the revision procedure, intraoperative findings, implants revised, incision length, and type of surgical approach used for the index TKA.

We determined differences in duration from index procedure to revision, age, and BMI using the Kruskal-Wallis rank test. A multivariate logistic regression was performed to evaluate the odds of having revision surgery less than 2 years from index TKA. Statistical analyses were performed with the SPSS (Chicago, Illinois) statistical software package.

\section{Results}

The average ages between the two groups were similar at the time of their primary TKA (57 years for MI TKA vs. 54 years for traditional TKA, $p=0.74$ ) and were similar at the time of their revision TKA (59 years for MI TKA vs. 60 years for traditional TKA, $p=0.89$ ). The primary diagnosis before the index procedure was osteoarthritis in $82 \%$ of the MI TKA patients and $92 \%$ of the traditional TKA patients. There was no difference in BMI between the two groups ( $34 \pm 5 \mathrm{~kg} / \mathrm{m}^{2}$ MI TKA vs. $33 \pm 10 \mathrm{~kg} / \mathrm{m}^{2}$ traditional TKA, $p=0.43$ ) (-Tables 1 and $\mathbf{2}$ ).

The non-MI TKA group had a longer time to revision compared with the MI TKA group (mean 65 months, range 7 to 175 months vs. mean 29 months, range 6 to 102 months, respectively, $p=0.032$ ). Of the 11 MI TKA patients, 8 (73\%) were revised within 24 months of their index procedure versus $15 \%(2 / 13)$ of the traditional TKA group $(p=0.005)$. When controlled for age, gender, and BMI, having a MI TKA conferred an increased risk of having revision surgery within 2 years of the index operation (odds ratio 14.7) (-Table 1).

Table 1 Comparison of MI and Non-MI TKA Failures

\begin{tabular}{|c|c|c|c|c|}
\hline Variable & MI TKA $(n=11)$ & Non-MI TKA $(n=13)$ & $p$ Value & OR \\
\hline Age at first TKA (years) & 57 (range, 50-63) & 54 (range, 42-69) & 0.74 & \\
\hline Gender (percent female) & $64 \%$ & $38 \%$ & 0.22 & \\
\hline $\mathrm{BMI}\left(\mathrm{kg} / \mathrm{m}^{2}\right)$ & 34 & 33 & 0.43 & \\
\hline Time to revision (months) & 28.6 (range, 6-102) & 64.7 (range, 7-175) & 0.032 & \\
\hline Percent revised $<2$ years after index procedure & $73 \%(8 / 11)$ & $15 \%(2 / 13)$ & 0.005 & 14.7 \\
\hline
\end{tabular}

BMI, body mass index; MI, minimally invasive; MI TKA, minimally invasive total knee arthroplasty; OR, odds ratio; TKA, total knee arthroplasty. 
Table 2 MI TKA Patient Characteristics

\begin{tabular}{|c|c|c|c|c|c|c|}
\hline $\mathrm{N}$ & Age at Primary (Years) & Gender & BMI $\left(\mathrm{kg} / \mathrm{m}^{2}\right)$ & Diagnosis & $\begin{array}{l}\text { Age at Revision } \\
\text { (Years) }\end{array}$ & $\begin{array}{l}\text { Time to } \\
\text { Revision (Months) }\end{array}$ \\
\hline 1 & 52 & $\mathrm{~F}$ & 38 & Psoriatic arthritis & 53 & 6 \\
\hline 2 & 63 & $\mathrm{~F}$ & 37 & $\mathrm{OA}$ & 65 & 19 \\
\hline 3 & 57 & $\mathrm{~F}$ & 37 & $\mathrm{OA}$ & 57 & 10 \\
\hline 4 & 61 & $\mathrm{~F}$ & 32 & $\mathrm{OA}$ & 62 & 18 \\
\hline 5 & 59 & $\mathrm{M}$ & 26 & $\mathrm{OA}$ & 62 & 39 \\
\hline 6 & 51 & $\mathrm{~F}$ & 35 & $\mathrm{OA}$ & 53 & 19 \\
\hline 7 & 60 & $\mathrm{M}$ & 33 & $\mathrm{OA}$ & 61 & 15 \\
\hline 8 & 50 & $\mathrm{~F}$ & 23 & Posttraumatic arthrosis & 51 & 16 \\
\hline 9 & 62 & $M$ & 37 & $\mathrm{OA}$ & 66 & 51 \\
\hline 10 & 59 & $M$ & 36 & $\mathrm{OA}$ & 60 & 20 \\
\hline 11 & 51 & $\mathrm{~F}$ & 40 & $\mathrm{OA}$ & 60 & 102 \\
\hline
\end{tabular}

BMI, body mass index; MI TKA, minimally invasive total knee arthroplasty; OA, osteoarthritis.

The reasons for revision in the traditional TKA group included aseptic loosening $(n=7)$, instability $(n=2)$, component malpositioning $(n=1)$, combined instability and component malpositioning $(n=1)$, polyethylene wear $(n=1)$, and pain/stiffness $(n=1)$. The reasons for revision in the MI TKA group included aseptic loosening $(n=6)$, pain/ stiffness $(n=3)$, instability $(n=1)$, and component malpositioning $(n=1)$ (-Table 3 ).

\section{Discussion}

We performed this analysis based on a growing concern that a disproportionate percentage of our aseptic revision TKA cases were performed for failed primary MI TKA. Evidence now suggests that MI techniques, when applied to total hip and knee arthroplasty, may be a cause of early failure. ${ }^{10,19}$ The data in this study yielded an association between MI TKA and the need for early revision surgery when compared with traditional TKA. Further, when controlled for age, gender, and BMI, having MI TKA conferred a 14-fold increased risk of having revision surgery within 2 years of the index operation. While aseptic loosening was the most common failure mechanism in both groups, the short-term failures may have been due to poor cement technique rather than polyethylene wear particle-mediated osteolysis.

There are several limitations of our present study. The first limitation is the small sample size. Although 11 failed MI TKAs over a 3-year period is a small case series, the scope of this problem may increase dramatically in the near future given the relatively recent adoption of MI techniques as well as the ongoing marketing campaigns produced by many surgeons. The second limitation is a poorly characterized referral population. Our tertiary joint replacement practice accepts referrals from the local and regional communities. Although

Table 3 Operative Data for MI TKA Patients

\begin{tabular}{|l|l|l|}
\hline $\mathbf{N}$ & Reason for Revision & Revision \\
\hline 1 & Pain, stiffness, retained cement & Liner \\
\hline 2 & Aseptic loosening & Femur \\
\hline 3 & Malrotation & Femur and tibia \\
\hline 4 & Aseptic loosening & Femur and tibia \\
\hline 5 & Aseptic loosening & Tibia \\
\hline 6 & Aseptic loosening & Femur and tibia \\
\hline 7 & Aseptic loosening & Femur and tibia \\
\hline 8 & Instability & Liner \\
\hline 9 & Stiffness & Femur and tibia \\
\hline 10 & Stiffness & Femur and tibia \\
\hline 11 & Aseptic loosening & Femur \\
\hline
\end{tabular}

MI TKA, minimally invasive total knee arthroplasty. 
most of these referrals were for septic processes or rerevision ( $59 \%$ in this series of total knee revisions), many of these cases are for aseptic failures. Without the benefit of a captured population as one might see within the Veterans Health Administration or a closed model health maintenance organization, it is impossible for us to characterize the population that makes referrals to our practice and the number of $\mathrm{MI}$ TKAs being performed within that group. Also, in the referral population seen at our academic medical center, we may be experiencing the learning curve of community orthopedic surgeons who are introducing these techniques to their practice. Authors have described learning curves of between 10 and 50 cases with the adoption of MI TKA techniques. ${ }^{20,21}$ The third limitation is that we chose a conservative definition of MI surgery as a skin incision $<11 \mathrm{~cm}$. The surgical approach was excluded from our analysis as was the perioperative rehabilitation protocol that the patient experienced during the primary TKA. The fourth limitation is that our data collection was subject to the accuracy of previous medical records and patient history.

MI techniques have been developed in attempts to improve functional recovery following TKA. Previous studies evaluating quadriceps strength following traditional TKA demonstrated a 17 to $24 \%$ deficit 1 year after surgery. ${ }^{22,23}$ This deficit was noted to persist and be detectable 6 to 13 years after surgery. ${ }^{22}$ Quadriceps weakness has been identified as one of the most important predictors of chronic disability following TKA. ${ }^{24-26}$ MI approaches (i.e., mini-midvastus, the subvastus, and mini-arthrotomy) limit disruption to the extensor mechanism, thereby decreasing quadriceps weakness. Through a mini-subvastus approach, Schroer et $\mathrm{al}^{27}$ noted a return of quadriceps strength to preoperative levels by 3 months postoperatively and that patients' quadriceps were $30 \%$ stronger than preoperative levels at 1 year. Improved quadriceps strength may allow patients to perform an independent straight leg raise, improve walking distances, and permit patients to discontinue the use of assistive devices earlier. However, a more limited exposure may place increased stress on bone surfaces, the skin, and pericapsular tissues. This has led to complications such as femoral condyle fracture, patellar tendon disruption, soft tissue invagination at interfaces, overresection of bone, retained extraneous cement, and component malposition. ${ }^{28,29}$ Opponents of MI TKA note that published literature comparing MI TKA and conventional TKA are underpowered, and are therefore incapable of reporting any meaningful statistical comparison of complication rates. Literature evaluating outcomes after MI TKA consists mainly of nonrandomized prospective and retrospective studies. The majority of the published data stem from developers of these techniques. Inclusion criteria are strict and may bias the observed outcomes. Gandhi et $\mathrm{al}^{30}$ recently published a meta-analysis comparing the incidence of complications between MI and traditional TKA. They noted a statistically significant increase in complication rates associated with MI techniques ( $p<0.04$ ), and no significant differences in postoperative radiographic alignment or function at 3 months postoperatively. Kolisek et $\mathrm{al}^{31}$ conducted a prospective, randomized, multicenter study to evaluate the safety and efficacy of the MI approach compared with conventional TKA. The authors found no statistically significant difference between the groups in terms of blood loss, operative time, infection, ultimate wound healing, postoperative pain, length of hospital stay, and functional outcome by 3 months postoperatively.

Middle and long-term outcome studies are now being published for MI TKA. Watanabe et al published a retrospective review comparing MI TKA to the conventional approach for TKA. ${ }^{32}$ They found a statistically significant improvement in the ability to perform a straight leg raise in the MI group. All other outcome measures were equivocal. They additionally noted that knee function was not significantly different between the groups at 2- to 4-year follow-up. Significant component misalignment occurred in three patients in the MI group and one patient in the control group. Varela-Egocheaga et $\mathrm{al}^{12}$ conducted a prospective, randomized trial comparing a MI subvastus approach to the conventional parapatellar approach. Exclusion criteria were broad and included knee flexion contractures greater than 10 degrees, varus/valgus angulation greater than 20 and 15 degrees, respectively, BMI greater than $40 \mathrm{~kg} / \mathrm{m}^{2}$, or those who had previous knee surgery. Length of follow-up was considered long-term at 3 years. Statistically significant findings in the MI group included increased perioperative drainage, less narcotic consumption, early time to walking, and improved ROM on postoperative day 3. ROM and the global Knee Society Score at 1 month, 3 months, 1 year, and 3 years after surgery were higher in the MI group. The authors concluded that early and late functional results were substantially better with a MI subvastus approach compared with the conventional approach.

When performed through the traditional approach, TKA remains one of the most successful orthopedic procedures. While surgeon satisfaction may exceed patient satisfaction, more than 75 to $89 \%$ of patients are satisfied with their outcomes. $^{3-5,33-36}$ Patient concerns regarding postoperative pain, length of recovery, and length of surgical incision have led to the creation of MI surgical techniques for TKA. The reliability of a TKA procedure performed through a small incision, and its success, may depend heavily on patient selection and surgeon experience. Long-term survivorship of traditional TKA should not be compromised for short-term gains espoused by MI TKA. The data from the current study corroborate the work of Barrack et $\mathrm{al}^{10}$ and suggest that minimal incision TKA may be a risk factor for early revision. Further study is needed to refine patient selection criteria and to evaluate the long-term outcomes of MI TKA.

\section{References}

1 Shankar NS. Minimally invasive technique in total knee arthroplasty-history, tips, tricks and pitfalls. Injury 2006;37(Suppl 5): S25-S30

2 Bullens PH, van Loon CJ, de Waal Malefijt MC, Laan RF, Veth RP. Patient satisfaction after total knee arthroplasty: a comparison between subjective and objective outcome assessments. J Arthroplasty $2001 ; 16(6): 740-747$ 
3 Vail TP. Minimally invasive knee arthroplasty. Clin Orthop Relat Res 2004;(428):51-52

4 Bourne RB, Chesworth BM, Davis AM, Mahomed NN, Charron KD. Patient satisfaction after total knee arthroplasty: who is satisfied and who is not? Clin Orthop Relat Res 2010;468(1):57-63

5 Noble PC, Conditt MA, Cook KF, Mathis KB. The John Insall Award: patient expectations affect satisfaction with total knee arthroplasty. Clin Orthop Relat Res 2006;452:35-43

6 Anderson JG, Wixson RL, Tsai D, Stulberg SD, Chang RW. Functional outcome and patient satisfaction in total knee patients over the age of 75. J Arthroplasty 1996;11(7):831-840

7 Heck DA, Robinson RL, Partridge CM, Lubitz RM, Freund DA. Patient outcomes after knee replacement. Clin Orthop Relat Res 1998;356(356):93-110

8 Robertsson O, Dunbar M, Pehrsson T, Knutson K, Lidgren L. Patient satisfaction after knee arthroplasty: a report on 27,372 knees operated on between 1981 and 1995 in Sweden. Acta Orthop Scand 2000;71(3):262-267

9 Repicci JA, Eberle RW. Minimally invasive surgical technique for unicondylar knee arthroplasty. J South Orthop Assoc 1999;8 (1):20-27, discussion 27

10 Barrack RL, Barnes CL, Burnett RS, Miller D, Clohisy JC, Maloney WJ. Minimal incision surgery as a risk factor for early failure of total knee arthroplasty. J Arthroplasty 2009;24(4):489-498

11 Tria AJ Jr. Advancements in minimally invasive total knee arthroplasty. Orthopedics 2003;26(8, Suppl):s859-s863

12 Varela-Egocheaga JR, Suárez-Suárez MA, Fernández-Villán M, González-Sastre V, Varela-Gómez JR, Rodríguez-Merchán C. Minimally invasive subvastus approach: improving the results of total knee arthroplasty: a prospective, randomized trial. Clin Orthop Relat Res 2010;468(5):1200-1208

13 Bonutti PM, Mont MA, McMahon M, Ragland PS, Kester M. Minimally invasive total knee arthroplasty. J Bone Joint Surg Am 2004;86-A(Suppl 2):26-32

14 Berger RA, Sanders S, Gerlinger T, Della Valle C, Jacobs JJ, Rosenberg AG. Outpatient total knee arthroplasty with a minimally invasive technique. J Arthroplasty 2005;20(7, Suppl 3):33-38

15 Huang HT, Su JY, Chang JK, Chen CH, Wang GJ. The early clinical outcome of minimally invasive quadriceps-sparing total knee arthroplasty: report of a 2-year follow-up. J Arthroplasty 2007;22(7): 1007-1012

16 Laskin RS, Beksac B, Phongjunakorn A, et al. Minimally invasive total knee replacement through a mini-midvastus incision: an outcome study. Clin Orthop Relat Res 2004;428(428):74-81

17 Tria AJJr, Coon TM. Minimal incision total knee arthroplasty: early experience. Clin Orthop Relat Res 2003;416(416):185-190

18 Callaghan JJ, Warth LC, Liu SS, Hozack WJ, Klein GR. Internet promotion of MIS and CAOS in TKA By Knee Society members. Clin Orthop Relat Res 2006;452:97-101

19 Graw BP, Woolson ST, Huddleston HG, Goodman SB, Huddleston JI. Minimal incision surgery as a risk factor for early failure of total hip arthroplasty. Clin Orthop Relat Res 2010;468(9):2372-2376

20 King J, Stamper DL, Schaad DC, Leopold SS. Minimally invasive total knee arthroplasty compared with traditional total knee arthroplasty. Assessment of the learning curve and the postoperative recuperative period. J Bone Joint Surg Am 2007;89(7): 1497-1503

21 Lubowitz JH, Sahasrabudhe A, Appleby D. Minimally invasive surgery in total knee arthroplasty: the learning curve. Orthopedics 2007;30(8, Suppl):80-82

22 Berman AT, Bosacco SJ, Israelite C. Evaluation of total knee arthroplasty using isokinetic testing. Clin Orthop Relat Res 1991;(271): 106-113

23 Rossi MD, Brown LE, Whitehurst M. Knee extensor and flexor torque characteristics before and after unilateral total knee arthroplasty. Am J Phys Med Rehabil 2006;85(9):737-746

24 Greene KA, Schurman JR II. Quadriceps muscle function in primary total knee arthroplasty. J Arthroplasty 2008;23(7, Suppl): 15-19

25 Mizner RL, Petterson SC, Snyder-Mackler L. Quadriceps strength and the time course of functional recovery after total knee arthroplasty. J Orthop Sports Phys Ther 2005;35(7):424-436

26 O'Reilly SC, Jones A, Muir KR, Doherty M. Quadriceps weakness in knee osteoarthritis: the effect on pain and disability. Ann Rheum Dis 1998;57(10):588-594

27 Schroer WC, Diesfeld PJ, Reedy ME, LeMarr AR. Isokinetic strength testing of minimally invasive total knee arthroplasty recovery. J Arthroplasty 2010;25(2):274-279

28 Aglietti P, Baldini A, Giron F, Sensi L. Minimally invasive total knee arthroplasty: is it for everybody? HSS J 2006;2(1):22-26

29 Dalury DF, Dennis DA. Mini-incision total knee arthroplasty can increase risk of component malalignment. Clin Orthop Relat Res 2005;440:77-81

30 Gandhi R, Smith H, Lefaivre KA, Davey JR, Mahomed NN. Complications after minimally invasive total knee arthroplasty as compared with traditional incision techniques: a meta-analysis. J Arthroplasty 2011;26(1):29-35

31 Kolisek FR, Bonutti PM, Hozack WJ, et al. Clinical experience using a minimally invasive surgical approach for total knee arthroplasty: early results of a prospective randomized study compared to a standard approach. J Arthroplasty 2007;22(1):8-13

32 Watanabe $T$, Muneta $T$, Ishizuki $M$. Is a minimally invasive approach superior to a conventional approach for total knee arthroplasty? Early outcome and 2- to 4-year follow-up. J Orthop Sci 2009;14(5):589-595

33 Laskin RS. Minimally invasive total knee arthroplasty: the results justify its use. Clin Orthop Relat Res 2005;440:54-59

34 Nilsdotter AK, Toksvig-Larsen S, Roos EM. Knee arthroplasty: are patients' expectations fulfilled? A prospective study of pain and function in 102 patients with 5-year follow-up. Acta Orthop 2009; 80(1):55-61

35 Rodríguez HA. Mini-incision total knee arthroplasty can increase risk of component malalignment. Clin Orthop Relat Res 2006;449:320, author reply 320

36 Vessely MB, Whaley AL, Harmsen WS, Schleck CD, Berry DJ. The Chitranjan Ranawat Award: long-term survivorship and failure modes of 1000 cemented condylar total knee arthroplasties. Clin Orthop Relat Res 2006;452:28-34 